\title{
Virtual Cell: a General \\ Framework for Simulating and Visualizing Cellular Physiology
}

\author{
Dong-Guk Shin and Lei Liu \\ Computer Science \& Engineering \\ University of Connecticut \\ 191 Auditorium Road, U-155, Storrs, CT 06269, USA, \\ \{shin, lei\}@eng2.uconn.edu \\ Leslie M. Loew and James Schaff \\ Center for Biomedical Imaging Technology \\ University of Connecticut Health Center \\ 253 Farmington Avenue, Farmington, CT 06030-1507, USA, \\ les@volt.uchc.edu,schaff@photon.uchc.edu
}

\begin{abstract}
The virtual cell project aims to develop a modeling framework that integrates cell structure and its associated functions. This framework poses two significant research challenges to computer scientists: (i) how to organize diversified sets of heterogeneous computational data so that the data can be seamlessly integrated into a computation model demonstrating complex cell biochemistry processes, and (ii) how to visually present, in a most intuitive fashion, the dynamics of cell physiology derived from the simulation model.
\end{abstract}

\section{Keywords}

Modeling of visual information, Visualization of simulation results

\section{INTRODUCTION}

We have been investigating development of a new computational framework called a "Virtual Cell." This computational tool allows scientists to observe and examine detailed steps of chemical and kinematic changes without conducting an actual experiment [3]. This could be useful if such changes cannot be observed in real laboratory experiment or if the cost of doing so is prohibitively high. This system requires exploitation of massive amount of spatial relationships involving cell structure components. It also involves a visualization component that is responsible for illustrating how a cell functions and interacts at the molecular level. Developing the virtual cell framework re- 


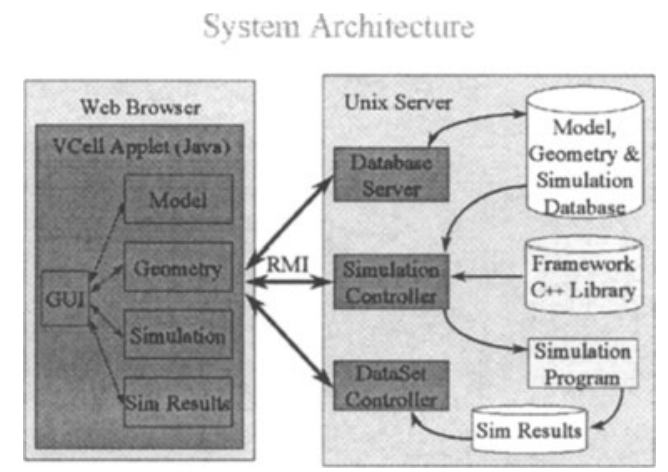

Figure 1 System Architecture

quires substantial computational resources. Due to the sheer amount of data and complexity, organizing these data sets requires a powerful database management system. Also, user interfaces are needed to facilitate various types of subtasks: creating and analyzing abstract models, generating specific simulations, controlling and monitoring simulations, and analyzing data.

\section{SYSTEM ARCHITECTURE}

The virtual cell framework is divided into two parts, the client and the server. Figure 1 shows an overview of the system architecture. The network communication between the client (Web Browser) and the server (Unix Sever) is done via Java RMI.

The client part is responsible for three major tasks: (i) creating and manipulating the abstract models (i.e., cellular physiological models), (ii) sending specifications and controlling the server side simulations, and (iii) analizing the results sent from the server simulation and displaying the analysis visually.

The server part maintains three types of data repositories: (a) Model, Geometry and Simulation (MGS) Database, (b) Simulation Result Database, and (c) Framework C++ Library. Our modular design for the disparate computing resources allows for an effective coordination of an arbitrary collection of variables, equations, problem solvers, and meshing techniques.

One challenge in the virtual cell database design is the heterogeneity among data models required for the system. Physiological models used in simulation processes are best described as objects. Each Model object consists of three components: species, reaction and feature. Species describe the molecules involved in simulation and their properties, such as their initial conditions. Reaction describes the biochemical process in terms of equations. Feature describes the spatial compartments within which the biochemical process takes 


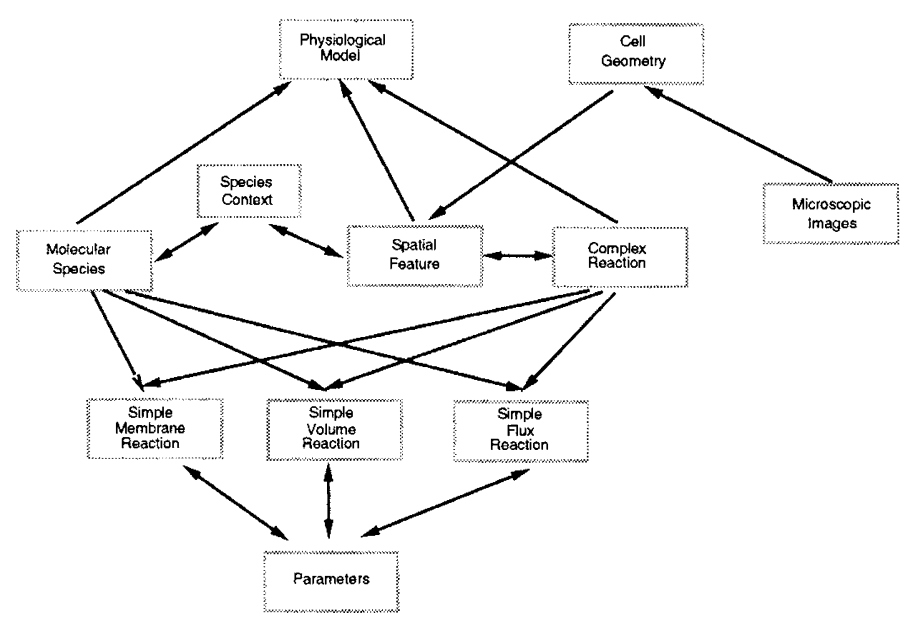

Figure 2 Virtual cell database design

place. Besides these three components, a set of quantitative parameters which describes the initial condition of each process have to be stored.

Another unique aspect of the virtual cell database is that the real microscopic images and the geometry derived from the real image are stored and related to the physiological models. In addition to querying the physiological models, the user should also be able to query the geometry of a particular type of cell.

\section{DATABASE DESIGN}

Figure 2 shows an abstract-level description of the relationships amongst the data used to produce a virtual cell model. Overall, there are four disparate sets of data.

Molecular Species Data include quantitative properties, such as initial concentrations and diffusion coefficients. They also include the location constraints, which indicate where in the cell the species exist. The species data need to be linked to spatial features, and such an association is modeled in terms of a relationship called "species context".

Spatial feature data describe the spatial relationships amongst various compartments of a cell. We note that the spatial relationships amongst cell compartments form a hierarchy that indicates whether or not a pair of cell compartments share boundaries. These hierarchical relationships need to be stored so that they can be used to visualize changes in cellular structure.

Biochemical Reaction Data are normally expressed in terms of diagrams involving complex chemical dynamics of various types of discrete particles. In order to simplify the design for a complex reaction, one often breaks down a complex reaction into a sequence of simpler reactions. Each simple reaction 


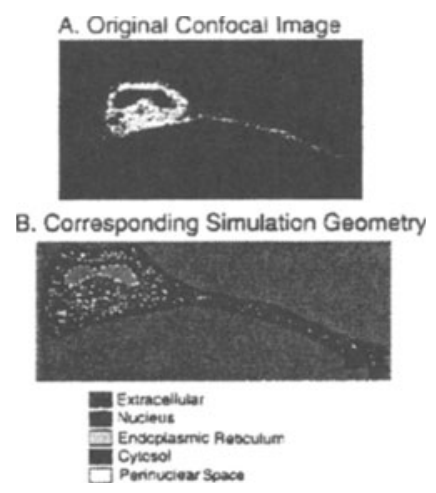

Figure 3 Cell geometry and microscopic image

is supposed to contain only one set of reactants, one set of products and one set of catalysts. The simple reactions are then categorized into three basic reactions according to the place where the reaction occur. Defining each simple reaction requires declaring molecular species as reactants, products or catalysts. In order to visualize the effects of reactions, some relationships that have been established between reactions and locations of spatial features should be used.

Cell geometry derived from microscopic image is another important component of the virtual cell. Figure 3 shows a derivation of a cell model for neuronal calcium signaling from a confocal micrograph of an ER labeled cell. Panel A in the figure represents an optical slice fully exhibited within both the neurite and soma. The ER has its highest density in the soma with a more diffuse distribution in the neurite and growth cone. The kidney shaped dark region in the soma is the nucleus. The image in Panel A was segmented and classified using simple thresholding techniques to define the indicted cellular and extracellular regions. The segmented image was then sampled at a lower pixel density to limit the mesh resolution for the simulation geometry. The spatial feature data (such as surface/volume ratio) is derived from the geometry.

\section{VISUALIZATION CONTROL USER INTERFACES}

These interfaces should present the physiology of electrochemical and structural phenomena in a natural and consistent manner. Three types of user interfaces can be built: one that edits abstract physiological models, one that edits abstract geometric description, and one that describes simulation constraints. For these interfaces to be consistent and maintainable, they should interact directly with the centrally maintained data sources. There are four sets of user interfaces through which users control parameters involved in 


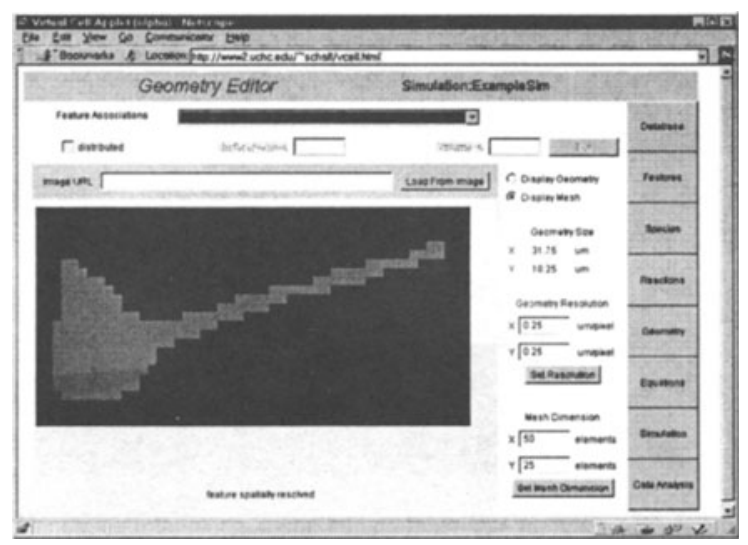

Figure 4 Geometry editor

displaying the virtual cell, namely, the species editor, the feature editor, the reaction editor, and the geometry editor. Figure 4 shows a snapshot of the geometry editor. Given an image retrieved from the image database, this editor allows the user to examine the image's geometry and to view the image in mesh format. One important function of this editor is that it allows the user to focus on features of the image which were a priori defined.

\section{LINKING TO OTHER DATABASES}

Currently we are at the stage of extending our present framework into a more advanced one. One goal of this next generation virtual cell framework is to link it to third party data resources that are available through the Internet. In particular, we plan to link our framework to two outside data sources - the cell image database and the metabolic pathway database. Figure 5 illustrates the future model.

Since virtual cell is a simulation of physiological processes, it is logical to have the virtual cell framework linked to a database which contains metabolic and regulatory pathway information. A database specializing in such information already exists and is called KEGG (Kyoto Encyclopedia of Genes and Genomes) [1]. In order to make use of KEGG, we plan to study: (i) how to derive and illustrate a diagram from the metabolic pathway database, and (ii) how to tie our simulation model with the diagrammatic presentation of the pathway information and visualize animation of reactions.

The second data source we plan to interlink with the virtual cell framework is a cell image database containing 2-D and 3-D geometries of cells. Once such candidate image database is the BioImage database which is being developed in Europe (e.g. Marabini et al. 1996 [2]).

Interlinking with the image database requires two research issues to be 


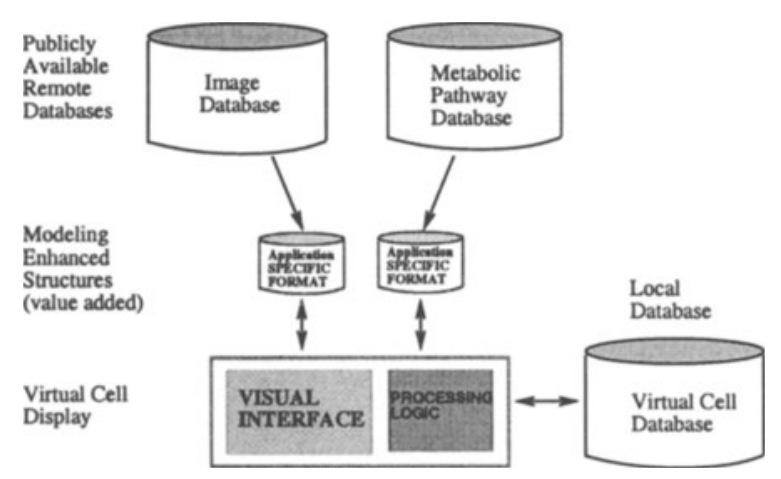

Figure 5 Interlinking with other databases

resolved: (i) how to convert the data in a format given by a third party database into a format that the virtual cell framework can handle, and (ii) how to resolve geometry incompatibility which can potentially occur when images from an outside database are imported and used with the existing internal models developed for different image geometry convention.

\section{CONCLUSION}

We described the virtual cell framework and its associated computational components. Much of the framework involves modeling and storing both conventional and unconventional data, developing user interfaces that allow users to refine experimental input parameters, and providing a mechanism for visualizing physiological reactions within the cell structure. The virtual cell exhibits a great potential as a tool capable of visualizing the dynamics of cell physiological processes. Once fully developed, we expect that the virtual cell can be very useful both as a research tool and a teaching aid.

\section{REFERENCES}

[1] M. Kanehisa. Toward pathway engineering: a new database of genetic and molecular pathways. In Science \& Technology Japan, volume 59, pages 34-38, 1996.

[2] R. Marabini, C. Vaquerizo, J.J. Fernandez, J.M. Carazo, A. Engel, and J. Frank. Proposal for a new distributed database of macromolecular and subcellular structures from different areas of microscopy. In $J$. Structural Biology, volume 116(1), pages 97-137, 1996.

[3] J. Schaff, C. C. Fink, B. Slepchenko, J. H. Carson, and L. M. Loew. A general computational framework for modeling cellular structure and function. In Biophys. J., volume 73, pages 1135-1146, 1997. 


\section{BIOGRAPHY}

Dong-Guk Shin is an associate professor in the Department of Computer Science and Engineering at the University of Connecticut. His research interests include graphical user interfaces for databases, database interoperability, and knowledge discovery from databases. Lei Liu is a post-doctoral fellow in the same department and specializes in cross-disciplinary research between Biology and Computer Science. Leslie Loew is a professor in the Department of Physiology at the University of Connecticut Health Center and is also the director of the Center for Biomedical Imaging Technology. James Schaff is a research associate at the Center for Biomedical Imaging Technology. 